\title{
Serum neurofilament light in familial Alzheimer disease
}

\author{
A marker of early neurodegeneration \\ OPEN
}

Philip S.J. Weston, MRCP

Teresa Poole, MSc Natalie S. Ryan, MRCP Akshay Nair, MRCPsych Yuying Liang, MRCP Kirsty Macpherson, BA Ronald Druyeh, BSc Ian B. Malone, PhD R. Laila Ahsan, MSc Hugh Pemberton, MSc Jana Klimova, MSc Simon Mead, PhD Kaj Blennow, MD Martin N. Rossor, MD Jonathan M. Schott, MD Henrik Zetterberg, MD* Nick C. Fox, MD*

Correspondence to

Dr. Fox:

n.fox@ucl.ac.uk

Editorial, page 2126

Supplemental data at Neurology.org

\section{ABSTRACT}

Objectives: To investigate whether serum neurofilament light (NfL) concentration is increased in familial Alzheimer disease (FAD), both pre and post symptom onset, and whether it is associated with markers of disease stage and severity.

Methods: We recruited 48 individuals from families with PSEN1 or APP mutations to a crosssectional study: 18 had symptomatic Alzheimer disease (AD) and 30 were asymptomatic but at $50 \%$ risk of carrying a mutation. Serum NfL was measured using an ultrasensitive immunoassay on the single molecule array (Simoa) platform. Cognitive testing and MRI were performed; 33 participants had serial MRI, allowing calculation of atrophy rates. Genetic testing established mutation status. A generalized least squares regression model was used to compare serum NfL among symptomatic mutation carriers, presymptomatic carriers, and noncarriers, adjusting for age and sex. Spearman coefficients assessed associations between serum NfL and (1) estimated years to/from symptom onset (EYO), (2) cognitive measures, and (3) MRI measures of atrophy.

Results: Nineteen of the asymptomatic participants were mutation carriers (mean EYO -9.6); 11 were noncarriers. Compared with noncarriers, serum NfL concentration was higher in both symptomatic ( $p<0.0001$ ) and presymptomatic mutation carriers ( $p=0.007$ ). Across all mutation carriers, serum NfL correlated with EYO ( $\rho=0.81, p<0.0001)$ and multiple cognitive and imaging measures, including Mini-Mental State Examination ( $\rho=-0.62, p=0.0001)$, Clinical Dementia Rating Scale sum of boxes $(\rho=0.79, p<0.0001)$, baseline brain volume $(\rho=-0.62$, $p=0.0002)$, and whole-brain atrophy rate $(\rho=0.53, p=0.01)$.

Conclusions: Serum NfL concentration is increased in FAD prior to symptom onset and correlates with measures of disease stage and severity. Serum NfL may thus be a feasible biomarker of early AD-related neurodegeneration. Neurology ${ }^{\circledR} 2017 ; 89: 2167-2175$

\section{GLOSSARY}

$\mathbf{A D}=$ Alzheimer disease; $\mathbf{A L S}=$ amyotrophic lateral sclerosis; $\mathbf{C D R}=$ Clinical Dementia Rating Scale; $\mathbf{C l}=$ confidence interval; EYO = estimated years from symptom onset; FAD = familial Alzheimer disease; FTD = frontotemporal dementia; $\mathbf{M C l}=$ mild cognitive impairment; MMSE = Mini-Mental State Examination; NART = National Adult Reading Test; NfL = neurofilament light; PSP = progressive supranuclear palsy; QC = quality control; RMT = Recognition Memory Test; Simoa = single molecule array; $\mathbf{S O B}=$ sum of boxes; TIV = total intracranial volume; WASI $=$ Wechsler Abbreviated Scale of Intelligence.

There is great interest in testing potential disease-modifying treatments for Alzheimer disease (AD) prior to onset of symptoms. To facilitate this, biomarkers are needed to identify at-risk individuals, stage their disease, and track disease progression. ${ }^{1}$ Ideally, such biomarkers should be noninvasive, inexpensive, and simple to acquire. ${ }^{2}$ Blood-based biomarkers would be very valuable but are more challenging than CSF measures for several reasons, including lower blood concentration of the target analyte, making reliable quantification more difficult. ${ }^{3}$

\footnotetext{
*These authors contributed equally to this work.

From the Dementia Research Centre (P.S.J.W., T.P., N.S.R., A.N., Y.L., K.M., I.B.M., R.L.A., H.P., J.K., M.N.R., J.M.S., N.C.F.) and MRC Prion Unit (R.D., S.M.), Department of Neurodegenerative Diseases, UCL Institute of Neurology; Department of Medical Statistics (T.P.), London School of Hygiene \& Tropical Medicine, UK; and Clinical Neurochemistry Laboratory, Institute of Neuroscience and Physiology (K.B., H.Z.), the Sahlgrenska Academy at the University of Gothenburg, Mölndal, Sweden.

Go to Neurology.org for full disclosures. Funding information and disclosures deemed relevant by the authors, if any, are provided at the end of the article. The Article Processing Charge was funded by Medical Research Council (UK).

This is an open access article distributed under the terms of the Creative Commons Attribution License 4.0 (CC BY), which permits unrestricted use, distribution, and reproduction in any medium, provided the original work is properly cited.
} 
One promising neurodegeneration biomarker in CSF is neurofilament light (NfL), which increases in a number of neurologic conditions, including $\mathrm{AD} \cdot{ }^{4-8} \mathrm{NfL}$ can be detected in serum using standard immunoassay formats, ${ }^{9,10}$ but many samples have concentrations below the analytical sensitivity of the methods. ${ }^{11}$ We therefore used a recently developed immunoassay based on the single molecule array $(\text { Simoa })^{12}$ that is 25 -fold as sensitive as the previous electrochemiluminescencebased method for NfL. ${ }^{11}$

We measured serum NfL concentrations in familial $\mathrm{AD}$ (FAD) mutation carriers and mutation-negative relatives. FAD shares many features, pathophysiologically and clinically, with the more common sporadic form of disease. ${ }^{13}$ FAD mutation carriers have relatively predictable ages at onset, ${ }^{14}$ which allows prospective study of individuals prior to onset of clinical AD. We hypothesized that, with a more sensitive assay, elevated serum NfL would be detectable in FAD mutation carriers prior to symptom onset, and would correlate with disease stage and rate of decline.

METHODS Standard protocol approvals, registrations, and patient consents. The study was approved by the local research ethics committee and all participants provided written informed consent.

Participants. We recruited 48 participants from 24 FAD families to a study at the Dementia Research Centre, University College London, between April 2010 and September 2015. Individuals were eligible if they had either a clinical diagnosis of FAD or a parent with FAD. Eighteen participants were symptomatic, with pathogenic mutations in the PSEN1 or APP genes; 30 individuals were asymptomatic but, by virtue of having an affected parent, were at $50 \%$ risk of having inherited a mutation and thereby of developing symptoms at a similar age to their parent (see table e-1 at Neurology.org for family mutations).

For all participants, genetic testing using Sanger sequencing determined the presence or absence of a mutation. Genetic data were provided only to statisticians, ensuring participants and clinicians remained blinded to genetic status; for this reason, it was not possible prospectively to match asymptomatic mutation carriers and noncarriers. Estimated years from symptom onset (EYO) was calculated for mutation carriers by subtracting the age at which the participant's affected parent first developed progressive cognitive symptoms from the participant's current age.

Study procedures included blood sampling, a semi-structured health questionnaire (including exclusion of recent head injury), neurologic examination, cognitive assessment, and volumetric brain MRI, with all assessments completed within 4 months of blood sample collection.

The study was approved by the Queen Square Research Ethics Committee and all participants provided written informed consent.
Cognitive assessment. Cognitive assessment included the Wechsler Abbreviated Scale of Intelligence (WASI), ${ }^{15}$ the National Adult Reading Test (NART) (a measure of premorbid IQ), ${ }^{16}$ Recognition Memory Test (RMT) for Faces and Words, ${ }^{17}$ and the Mini-Mental State Examination (MMSE). A close informant was interviewed separately to obtain a collateral history. The Clinical Dementia Rating Scale $(\mathrm{CDR})^{18}$ provided an additional estimate of clinical severity; both global CDR and $\mathrm{CDR}$ sum of boxes (SOB) were calculated. Individuals were defined as symptomatic if global CDR was $>0$ and consistent symptoms of cognitive decline were reported by the participant or an informant.

Measurement of serum NfL concentrations. Serum samples were collected, processed, aliquoted, and frozen at $-80^{\circ} \mathrm{C}$ according to standardized procedures. We measured serum NfL using an ultrasensitive immunoassay on the Simoa platform, using the same methodology as described previously. ${ }^{19}$ The lower limits of detection and quantification, as defined by the concentration derived from the signal of blank samples (sample diluent) +3 and $10 \mathrm{SDs}$, were 0.97 and $2.93 \mathrm{pg} / \mathrm{mL}$, respectively. For a quality control (QC) sample with a concentration of $13.0 \mathrm{pg} / \mathrm{mL}$, repeatability was $14.0 \%$ and intermediate precision was $15.7 \%$. For a QC sample with a concentration of $131.8 \mathrm{pg} / \mathrm{mL}$, repeatability was $13.3 \%$ and intermediate precision was $13.3 \%$. All measurements were performed by board-certified laboratory technicians in one round of experiments using one batch of reagents.

MRI acquisition and analysis. MRI was obtained on 43 of the 48 participants at the time of the blood sample. Five participants were not scanned due to either declining or an inability to tolerate the scan (e.g., claustrophobia). For 33 of the 43 participants with an initial scan, a second scan was performed at a separate visit (mean interval $\pm \mathrm{SD}=1.3 \pm 0.46$ years); the other 10 individuals had no second scan due to either leaving the study $(\mathrm{n}=5)$ or it finishing before their second scan date $(n=5)$.

All scans were performed on the same 3T Siemens (Munich, Germany) TIM Trio scanner using a 32-channel phased array head coil. A sagittal 3D magnetization-prepared rapid gradient echo T1-weighted volumetric MRI (echo time/repetition time/ inversion time $=2.9 / 2,200 / 900 \mathrm{~ms}$, dimensions $256 \times 256 \times$ 208 , voxel size $1.1 \times 1.1 \times 1.1 \mathrm{~mm}$ ) was acquired. Images were visually checked for artifacts. Four baseline scans and 2 follow-up scans were excluded due to either movement or metallic dental artifact, leaving 39 scans available for baseline volume measurements and 30 pairs of scans for rates of atrophy measurements. Whole brain, ventricular, and hippocampal volumes were calculated using semiautomated methods. ${ }^{20}$ For ventricles and hippocampi, the mean volume from right and left hemispheres was calculated. All volumes were corrected for total intracranial volume (TIV) by dividing a participant's volume by TIV and multiplying by the group mean TIV. Annualized rates of brain, ventricular, and hippocampal volume change during the interscan interval were calculated using the boundary shift interval, a registration-based measure of within-subject volume change. ${ }^{21}$

Statistical analysis. The primary objective of the study was to compare serum NfL among symptomatic mutation carriers, presymptomatic mutation carriers, and noncarrier controls. A generalized least squares linear regression model, an extension of the $t$ test/analysis of variance model that allows different group-specific residual variances, was used to compare NfL between groups, adjusting for age and sex. Family was included as a random effect to assess any effect of clustering within a family.

Spearman correlation coefficients were calculated to assess the association between NfL and EYO, first across all mutation 
carriers and then in presymptomatic carriers only and symptomatic carriers only. This rank-based approach, which can be used with bounded variables and is robust to non-normality and outliers, was also used for NfL and cognitive measures, including estimated change in IQ (WASI IQ minus NART-predicted premorbid IQ), recognition memory (an average of scores from RMT faces and RMT words), MMSE, and CDR SOB. We also assessed associations between NfL and the MRI measures. For each association, we first calculated the Spearman coefficient using all available data points, and second using data only from individuals who completed all assessments.

We calculated Spearman correlation coefficients between EYO and each cognitive and imaging measure, including only presymptomatic participants. To allow results to be comparable, these analyses were done using only individuals with all available data points. For all analyses, missing values were assumed to be missing completely at random. Throughout, the threshold for statistical significance was set at $p<0.05$ (2-tailed) and no adjustment was made for multiple testing.

RESULTS Participants' demographic details, cognitive scores, neuroimaging measures, and serum NfL values are shown in table 1 and figure 1 . Of the asymptomatic participants, 19 were mutation carriers and 11 were noncarriers; noncarriers were used as healthy controls. The mean EYO of the presymptomatic mutation carriers was -9.6 years. Adjusting for age and sex, serum NfL concentration was significantly higher in symptomatic mutation carriers compared with presymptomatic mutation carriers (estimated difference in means $23.2 \mathrm{pg} / \mathrm{mL}, 95 \%$ confidence interval $[\mathrm{CI}]$ 13.1-33.2; $p<0.0001$ ) and with noncarriers $(29.2 \mathrm{pg} / \mathrm{mL}, 19.3-39.1 ; p<$ 0.0001). Presymptomatic mutation carriers had significantly higher NfL concentrations than noncarriers $(6.1 \mathrm{pg} / \mathrm{mL}, 1.6-10.5, p=0.007)$. Allowing for clustering within a family had no effect on results.

Across all mutation carriers, there was evidence of an association between serum NfL concentrations and EYO (Spearman $\rho=0.81, p<0.0001$ ), with individuals at a later disease stage having higher $\mathrm{NfL}$ concentrations (figure 2). Furthermore, this association was significant separately for both the presymptomatic $(\rho=0.55, p=0.01)$ and symptomatic $(\rho=$ $0.49, p=0.04)$ groups. A post hoc linear regression analysis in mutation carriers found no statistically significant association between NfL and age, after adjusting for EYO (i.e., disease stage) $(p=0.15)$.

Figure 3 shows scatterplots and Spearman coefficients for serum NfL against cognitive and imaging measures for all mutation carriers, with NfL concentration showing a relatively even distribution throughout the spectrum of disease severity. There

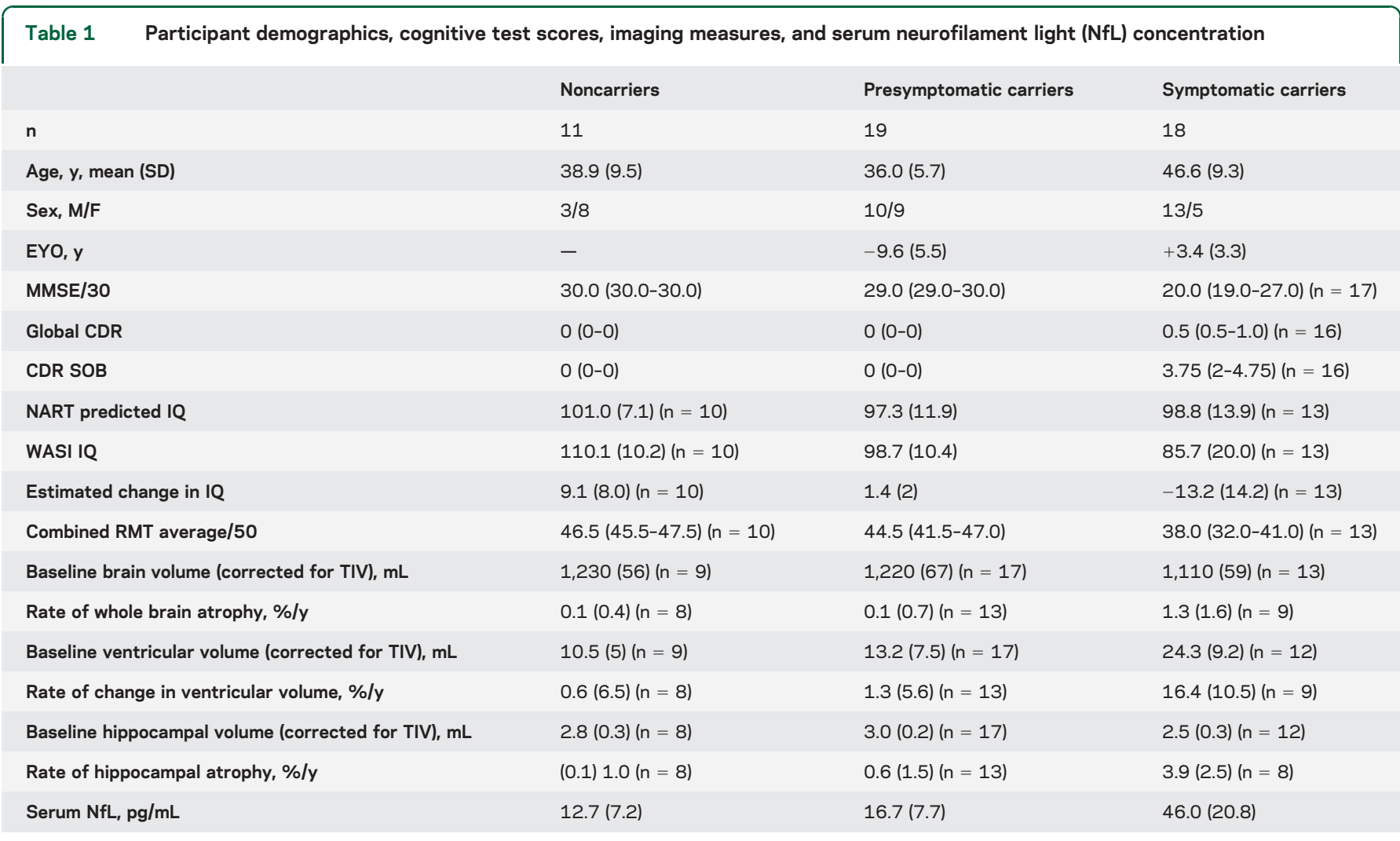

Abbreviations: $\mathrm{CDR}=$ Clinical Dementia Rating Scale; EYO = estimated years from onset; MMSE = Mini-Mental State Examination; NART = National Adult Reading Test; RMT = Recognition Memory Test; SOB = sum of boxes; TIV = total intracranial volume; WASI = Wechsler Abbreviated Scale of Intelligence. Estimated change in IQ was calculated by subtracting the current IQ (measured by the WASI) from the predicted premorbid IQ (measured by the NART). All values are group means (with SD), except for constrained variables (MMSE, global CDR, CDR SOB, and combined RMT), which are shown as median (interquartile range). Measures are uncorrected for any covariables. For variables with missing data points, the number of observations is shown after the group average value (e.g., $\mathrm{n}=\mathrm{x}$ ). 
Figure 1 Box and whisker plots for serum neurofilament light (NfL) across the 3 groups

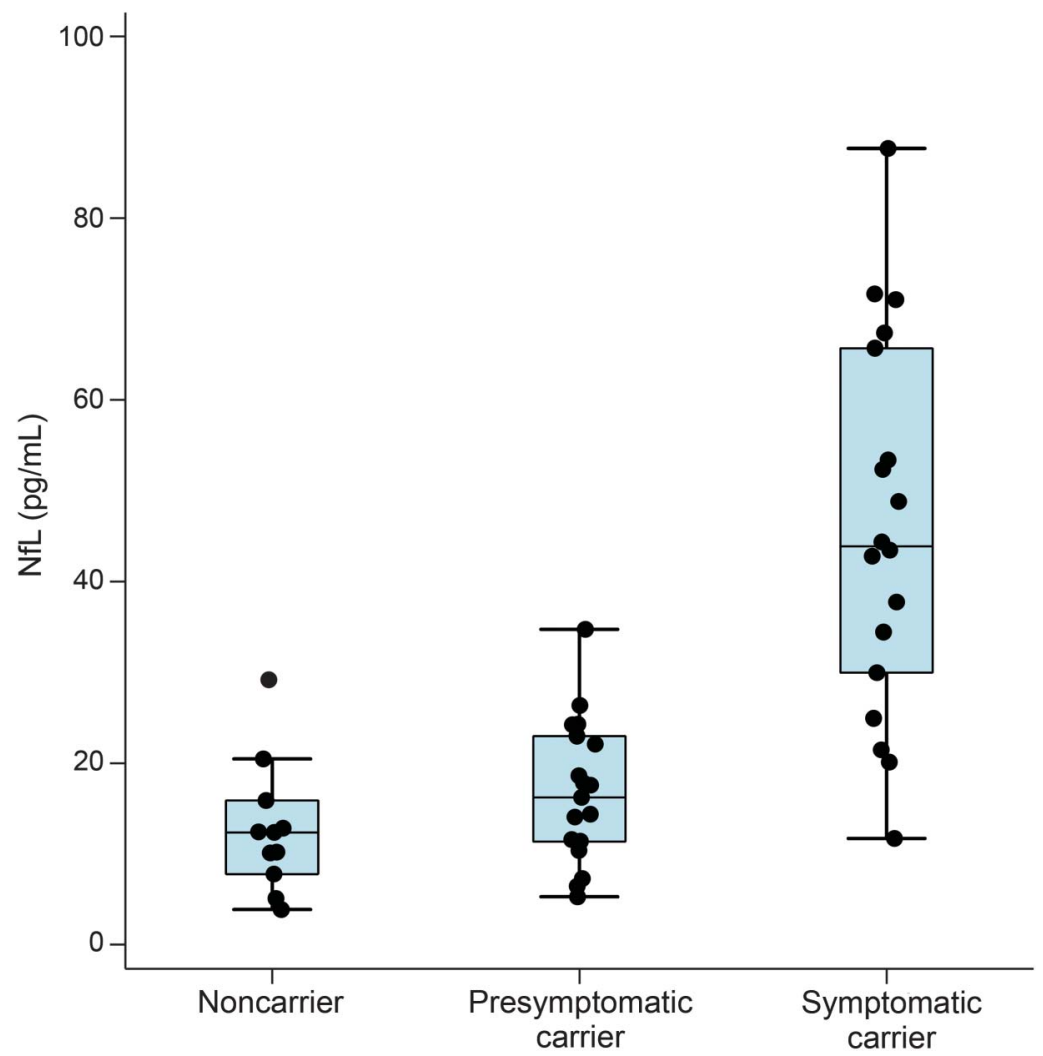

The measured unadjusted serum NfL concentrations are shown. Mutation carriers have been divided into those who are symptomatic and those who are presymptomatic.

were statistically significant correlations between serum NfL and cognitive measures, including MMSE, CDR SOB, and estimated change in IQ, with weaker evidence for a correlation with recognition memory score. In mutation carriers, there was a significant correlation between NfL and crosssectional neuroimaging measures, including baseline brain volume, baseline ventricular volume, and baseline hippocampal volume. There was also a significant correlation between serum NfL and subsequent rate of change in both brain volume and ventricular volume, but not hippocampal volume. Repeating the analysis including data only from the 19 individuals who completed all assessments did not lead to material change in results, other than for NfL and combined RMT ( $p$ value changed from 0.06 to 0.6 ).

For presymptomatic carriers only, there was weak evidence of a correlation between NfL and baseline ventricular volume $(\rho=0.43, p=0.08)$ and between $\mathrm{NfL}$ and CDR SOB ( $\rho=0.40, p=0.08)$, but no evidence of correlations with any other neuroimaging or cognitive measures.

When including only the 13 presymptomatic individuals with serial imaging, there remained a significant correlation between serum NfL and EYO (table 2). However, when assessing the correlations between each of the 6 imaging measures and EYO in the same individuals, none was statistically significant.

DISCUSSION Using an ultrasensitive immunoassay, we found serum NfL concentrations are increased in a group of symptomatic FAD mutation carriers who on average are only mildly clinically affected (median global CDR 0.5); we also found increased NfL concentrations in presymptomatic mutation carriers, who were on average 9 years from their predicted symptom onset. Serum NfL correlated significantly with the estimated years to/from symptom onset (EYO) across all mutation carriers, as well as in the symptomatic and presymptomatic groups separately.

Across all carriers, serum NfL correlated with CDR SOB and several cognitive measures. There was also a correlation between serum NfL and MRI measures of AD-related neurodegeneration, both in terms of cross-sectional volume loss and subsequent rates of atrophy. This suggests serum NfL concentrations may relate to disease severity or rate of progression.

Our serum NfL concentrations for symptomatic FAD are similar to a recent study of sporadic $A D$ that used the same ultrasensitive immunoassay approach. ${ }^{22}$ The mean concentration for our symptomatic group $(46.0 \mathrm{pg} / \mathrm{mL})$ (which contained a mixture of mild cognitive impairment $[\mathrm{MCI}]$ and $\mathrm{AD}$ dementia) lies between their mean values for separate sporadic MCI ( $42.8 \mathrm{pg} / \mathrm{mL})$ and AD dementia (51.0 $\mathrm{pg} / \mathrm{mL}$ ) groups. However, here we extend previous findings by showing that measurable increases in serum NfL precede the onset of symptomatic disease, and are correlated with predicted time to symptom onset. The observed progressive presymptomatic rise is consistent with proposed models of presymptomatic $\mathrm{AD}$ neurodegeneration. ${ }^{23} \mathrm{NfL}$ forms an important part of axonal structural integrity, with its rise likely to reflect early axonal breakdown. ${ }^{24}$

Our finding of a presymptomatic increase in serum NfL in FAD mutation carriers contrasts with findings from familial amyotrophic lateral sclerosis (ALS), where no increase was detected until after symptom onset despite symptomatic ALS participants having much higher concentrations than has been detected in either familial or sporadic AD. ${ }^{25}$ This likely reflects differences in the underlying biology and temporal pattern of neurodegeneration in $\mathrm{AD}$ vs ALS. ALS is a more aggressive neurodegenerative process in the symptomatic stage, but without the long, gradually progressive presymptomatic phase characteristic of AD. Importantly, atrophy rates are raised in the 5 years before symptoms in FAD and amyloid deposition appears even earlier. ${ }^{26-28}$ 
Figure 2 Scatterplot of serum neurofilament light $(\mathrm{NfL})$ against estimated years from symptom onset (EYO)

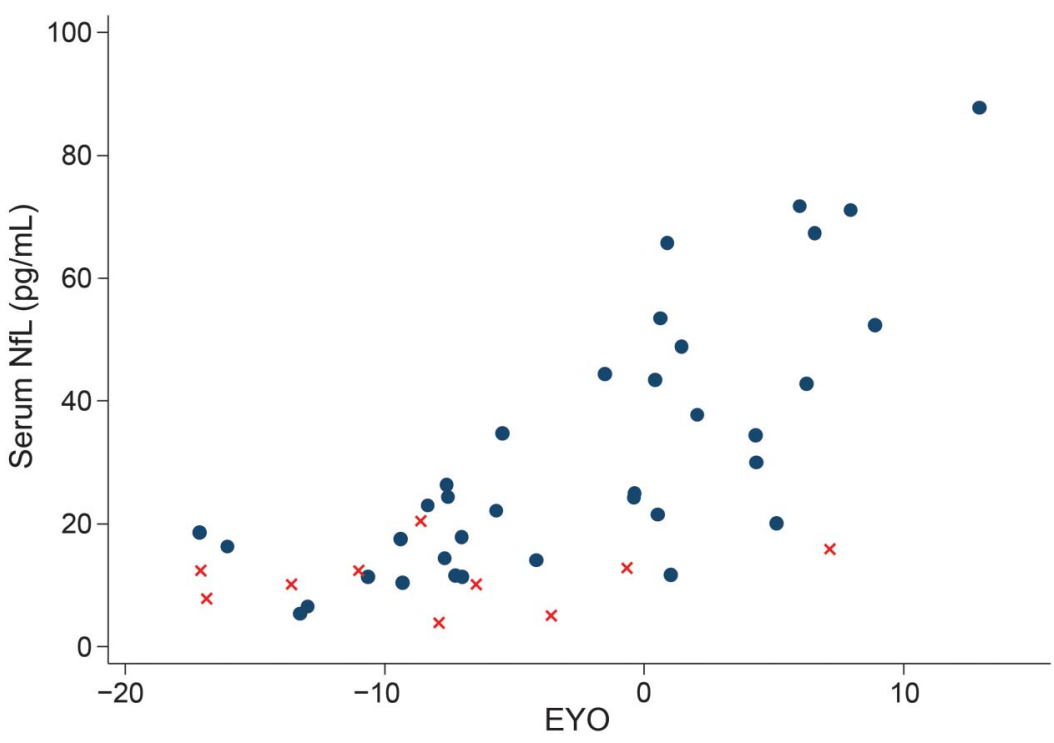

Mutation carriers are represented by dots and noncarriers by crosses. To ensure it is not possible to identify any of the individual asymptomatic participants (based on their EYO) and so determine their mutation status, 2 outlying participants have been removed and a jitter of up to \pm 2 years has been applied to all remaining participants.

The correlation of serum NfL with cognitive measures known to be sensitive to $\mathrm{AD}$-related decline supports the clinical relevance of NfL. While early cognitive changes in FAD most commonly involve episodic memory, ${ }^{29}$ we found that serum NfL correlated more strongly with global cognitive measures than with memory scores. This may relate to the physiologic role of NfL throughout the brain as an essential component of axonal stability, with initial rise possibly reflecting subtle widespread breakdown of neural networks, rather than focal, hippocampal (gray matter) atrophy. The possibility that elevated serum NfL levels more closely reflect global neurodegeneration is also supported by its correlation, across all carriers, with whole brain and ventricular volume loss. This contrasts with findings in other neurodegenerative diseases, including progressive supranuclear palsy (PSP) and frontotemporal dementia (FTD), where disease-specific focal atrophy appeared to be more strongly associated with NfL than whole brain atrophy. ${ }^{19,30}$

It is notable that, while serum NfL correlated significantly with disease stage (i.e., EYO) even when including only presymptomatic participants, imaging and cognitive measures did not. Serum NfL may therefore be a more sensitive marker of early neurodegeneration.

When measured in the CSF of individuals with MCI, NfL has been found to be predictive of subsequent progression to $\mathrm{AD}$ dementia, ${ }^{4}$ with a recent meta-analysis showing it to have comparable discriminatory power to the well-established CSF $\mathrm{AD}$ biomarkers of $A \beta_{1-42}$, total tau, and phosphorylated tau. ${ }^{3}$ Recent studies comparing NfL measurement in CSF and serum have shown close correlation, ${ }^{9,10,22}$ implying serum NfL may similarly predict subsequent progression, in keeping with our results.

A study in a FAD mouse model, which knocked out the NfL gene, showed that NfL deficiency significantly increased AD-related neurodegeneration, a finding that might suggest a role for NfL in maintaining neuronal structure in patients with $\mathrm{AD} .^{31}$ Moreover, in APP/PS1 mice, histopathologic examination found NfL-positive neuritic abnormalities, consistent with increased $\mathrm{NfL}$ in $\mathrm{AD}$, signifying underlying axonal damage. ${ }^{10}$ The same study showed serum NfL concentrations increased early in the disease and were closely associated with progression of AD-like pathology. Serum NfL concentrations decreased in response to anti-A $\beta$ immunotherapy, the authors suggesting that serum NfL may serve as a biomarker of treatment response.

There are obvious benefits to identifying $\mathrm{AD}$ biomarkers in blood, ${ }^{2}$ with numerous candidates proposed. ${ }^{32}$ However, recent comprehensive metaanalyses of blood-based markers showed only total tau reliably differentiated $\mathrm{AD}$ from healthy controls. ${ }^{3,33}$ Moreover, blood tau has only proven useful in identifying $\mathrm{AD}$ in established dementia cases, with no evidence that it is useful in earlier disease, and there is often overlap between patient and control groups. ${ }^{3,32}$ Studies attempting to measure blood concentrations of $A \beta_{1-42}$, the other core molecular marker of $\mathrm{AD}$ pathology, have so far produced conflicting results, with no strong overall evidence of a difference between $\mathrm{AD}$ and controls. ${ }^{3,32}$ Furthermore, even if $\beta$-amyloid moieties could be reliably identified and quantified, as cerebral $A \beta$ deposition is thought to plateau some time before symptom onset, ${ }^{28}$ it may not track progression unless very early in disease. By contrast, a marker of downstream neurodegeneration, such as NfL, which may reflect ongoing (global) disease activity, might be useful as a trial outcome measure, from presymptomatic to symptomatic phases. A blood test for neurodegeneration might also be useful clinically in identifying which individuals with cognitive concerns to prioritize for more detailed investigation.

A number of studies investigated plasma or serum profiles in an attempt to identify a pathologic fingerprint of $\mathrm{AD}$, using profiling approaches including proteomics, lipidomics, and transcriptomics. ${ }^{34-36}$ However, poor reproducibility remains an issue when assessing large panels of molecules involved in potentially diverse biological pathways, with several followon studies showing negative results. ${ }^{37-39}$ Although our 
Figure 3 Scatterplots of serum neurofilament light (NfL) against cognitive and imaging measures across all mutation carriers
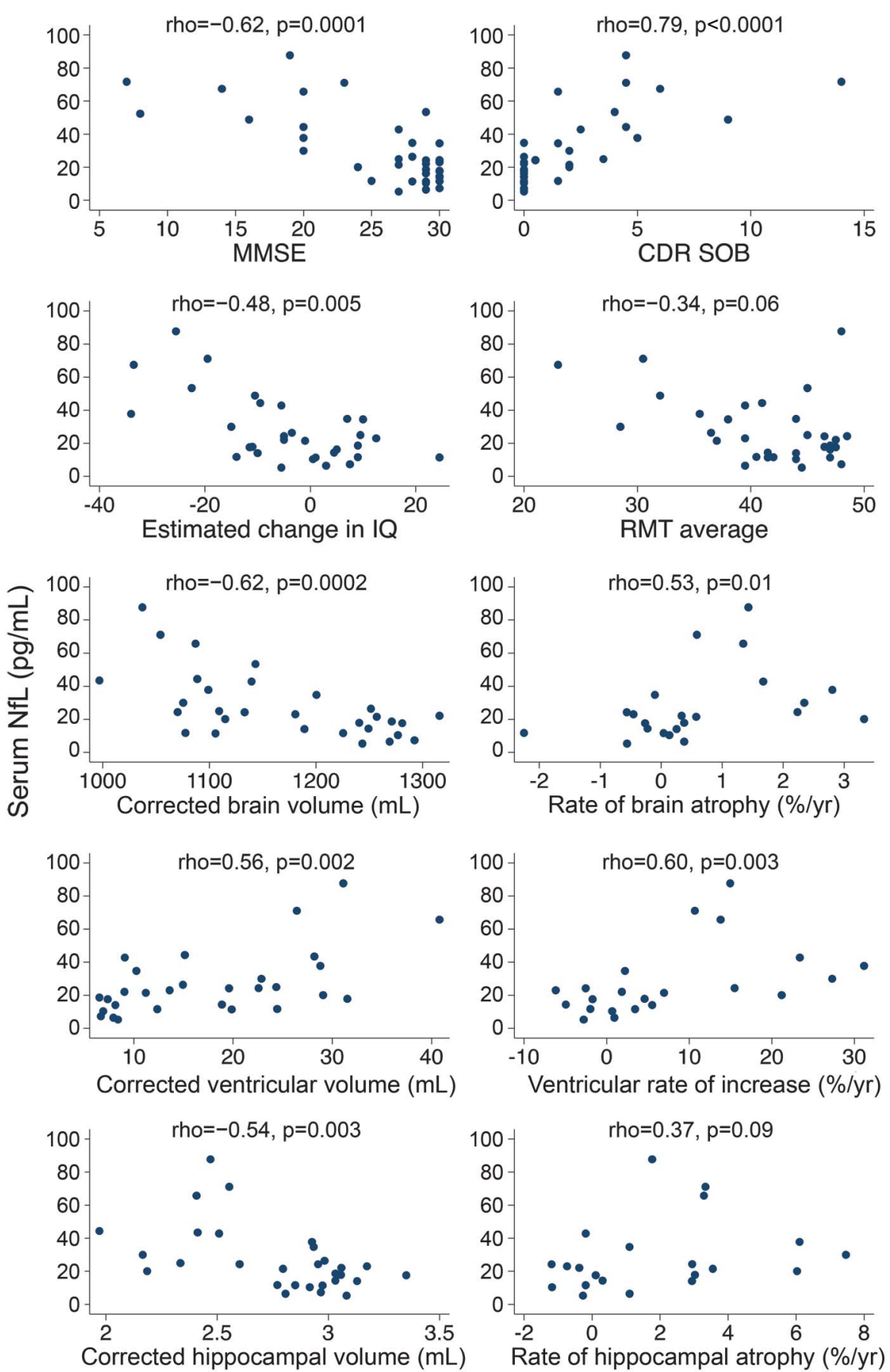

Spearman $\rho$ and the associated $p$ value are shown for each scatterplot. Estimated change in IQ was calculated by subtracting the current IQ (measured by the Wechsler Abbreviated Scale of Intelligence) from the predicted premorbid IQ (measured by the National Adult Reading Test). CDR SOB = Clinical Dementia Rating Scale sum of boxes; MMSE = MiniMental State Examination; RMT = Recognition Memory Test.

findings find support from previous studies of serum NfL in symptomatic $\mathrm{AD}, 9,10,22$ it will be important (1) to replicate the presymptomatic findings, before now shown only in mice, ${ }^{10}$ in other at-risk FAD and sporadic $\mathrm{AD}$ cohorts (e.g., in amyloid-positive older controls); and (2) to determine the clinical outcomes in these individuals to assess the predictive value and time course of increases in serum NfL.

While our results are encouraging, there are a number of issues regarding the utility of NfL as a biomarker of early $\mathrm{AD}$. While as a group the presymptomatic carriers had higher mean NfL than noncarriers, there was a degree of overlap in observed 
Table 2 Presymptomatic correlations between biomarkers and estimated years from symptom onset (EYO)

$\begin{array}{lcl}\text { Correlation between EYO and: } & \text { Spearman } \rho & p \text { Value } \\ \text { Serum NfL } & 0.73 & 0.005 \\ \text { MMSE } & 0.09 & 0.8 \\ \text { CDR SOB } & 0.51 & 0.07 \\ \text { Estimated change in IQ } & -0.23 & 0.5 \\ \text { Average RMT } & 0.52 & 0.07 \\ \text { Baseline brain volume (corrected for TIV), mL } & -0.43 & 0.1 \\ \text { Rate of whole brain atrophy, \%/y } & 0.09 & 0.8 \\ \text { Baseline ventricular volume, (corrected for TIV), mL } & 0.36 & 0.2 \\ \text { Rate of change in ventricular volume, \%/ y } & 0.52 & 0.07 \\ \text { Baseline hippocampal volume (corrected for TIV), mL } & -0.07 & 0.8 \\ \text { Rate of hippocampal atrophy, \%/y } & 0.18 & 0.6\end{array}$

Abbreviations: CDR SOB $=$ Clinical Dementia Rating Scale sum of boxes; MMSE $=$ MiniMental State Examination; NfL = neurofilament light; RMT = Recognition Memory Test; TIV $=$ total intracranial volume.

Estimated change in IQ was calculated by subtracting the current IQ (measured by the Wechsler Abbreviated Scale of Intelligence) from the predicted premorbid IQ (measured by the National Adult Reading Test). Spearman correlation coefficients for serum NfL, cognitive test scores, and imaging measures against EYO, in presymptomatic participants only. To allow for direct comparisons between the different measures, only the 13 presymptomatic mutation carriers for whom serum $\mathrm{NfL}$ and serial imaging measures were available are included.

values. The utility of serum NfL to diagnose presymptomatic $\mathrm{AD}$ at the individual level therefore remains uncertain and needs reassessment in independent cohorts. The changes in serum NfL through the course of the disease were analyzed in cross-sectional data only, so it is also not known whether serum NfL tracks progression at an individual level. Also, while our findings support the use of serum NfL as a marker of neurodegeneration in $\mathrm{AD}, \mathrm{NfL}$ is not a specific marker to $\mathrm{AD}$ and has been shown to increase in a number of other conditions, including HIV-associated dementia, PSP, FTD, and ALS. ${ }^{19,25,30,40}$ It may therefore be that serum NfL will be most useful for identifying and tracking ADrelated neurodegeneration when combined with a test to confirm underlying $\mathrm{AD}$ molecular pathology, e.g., CSF tau/A $\beta_{1-42}$ or amyloid PET.

Our study has limitations. The sample size was not large, owing primarily to the relative rarity of FAD mutations. However, this remains one of the largest single-center FAD cohorts yet reported. For a number of participants, not all cognitive and imaging assessments were completed. However, minimal changes were seen when rerunning the analyses to include only those participants who had completed all assessments. We estimated the age when each mutation carrier would be expected to develop symptoms based on parental age at onset, which is closely associated with actual age at onset ${ }^{14}$; however, this remains a proxy measure, and it is only with longitudinal follow-up that age at onset can be confirmed.

We show, using an ultrasensitive assay, that serum $\mathrm{NfL}$ concentration is increased in FAD prior to symptomatic disease, and correlates with the number of years to/from predicted symptom onset. Serum NfL also correlated with neuroimaging and cognitive markers of disease severity. Our findings support the further investigation of serum NfL as an easily accessible biomarker of early AD-related neurodegeneration.

\section{AUTHOR CONTRIBUTIONS}

P. Weston contributed to the conceptualization and design of the study, collection, analysis, and interpretation of the data, and drafting/revising of the manuscript. T. Poole led the statistical analysis and interpretation of the data and assisted in the drafting/revising of the manuscript. N. Ryan contributed to the collection of the data and drafting/revising of the manuscript. A. Nair contributed to the interpretation of the data and drafting/revising of the manuscript. Y. Liang contributed to the collection of the data and drafting/revising of the manuscript. K. Macpherson contributed to the collection of the data and drafting/revising of the manuscript. R. Druyeh contributed to the analysis of serum samples and drafting/revising of the manuscript. I. Malone contributed to the imaging analysis and drafting/revising of the manuscript. L. Ahsan contributed to the imaging analysis and drafting/revising of the manuscript. H. Pemberton contributed to the imaging analysis and drafting/revising of the manuscript. J. Klimova contributed to the imaging analysis and drafting/ revising of the manuscript. S. Mead contributed to the analysis of serum samples, interpretation of the data, and drafting/revising of the manuscript. K. Blennow contributed to the analysis of serum samples, interpretation of the data, and drafting/revising of the manuscript. M. Rossor contributed to the interpretation of the data and drafting/revising of the manuscript. J. Schott contributed to the interpretation of the data and drafting/revising of the manuscript. H. Zetterberg contributed to the conceptualization and design of the study, analysis of serum samples and interpretation of results, and drafting/revising of the manuscript. N. Fox contributed to the conceptualization and design of the study, interpretation of results, and drafting/revising of the manuscript.

\section{ACKNOWLEDGMENT}

The authors thank the participants and their families for their participation and Professor Chris Frost for his advice regarding the statistical analysis.

\section{STUDY FUNDING}

P.S.J.W. is supported by an MRC Clinical Research Training Fellowship. N.S.R. is supported by a Brain Exit Fellowship. A.N. is supported by a Wolfson Foundation fellowship. S.M. acknowledges support from the MRC and the NIHR Queen Square Dementia Biomedical Research Unit (BRU). K.B. receives support from the Torsten Söderberg Foundation at the Swedish Royal Academy of Sciences, the Research Council Sweden, the Brain Foundation Sweden, and the Alzheimer Foundation Sweden. M.N.R. and N.C.F. are NIHR Senior Investigators. The Dementia Research Centre is an ARUK Coordinating Centre and is grateful for support from the NIHR Queen Square Dementia BRU, the MRC Dementias Platform UK, and the Leonard Wolfson Experimental Neurology Centre. The study was undertaken at UCLH/UCL, which received a proportion of funding from the Department of Health's NIHR Biomedical Research Centres funding scheme. H.Z. is a Wallenberg Academy Fellow and receives support from the European Research Council, the Swedish Research Council, Frimurarestiftelsen, and VINNOVA.

\section{DISCLOSURE}

P. Weston, T. Poole, N. Ryan, A. Nair, Y. Liang, K. Macpherson, R. Druyeh, I. Malone, L. Ahsan, H. Pemberton, J. Klimova, and S. Mead report no disclosures relevant to the manuscript. K. Blennow has served as a consultant or at advisory boards for Alzheon, Eli Lilly, Fujirebio 
Europe, IBL International, Novartis, and Roche Diagnostics, and is a cofounder of Brain Biomarker Solutions in Gothenburg AB, a GU Venturebased platform company at the University of Gothenburg. M. Rossor reports no disclosures relevant to the manuscript. J. Schott has received research funding from AVID Radiopharmaceuticals (a wholly owned subsidiary of Eli Lilly), has consulted for Roche Pharmaceuticals and Eli Lilly, and serves on a Data Safety Monitoring Committee for Axon Neuroscience SE. H. Zetterberg is a co-founder of Brain Biomarker Solutions in Gothenburg AB, a GU Venture-based platform company at the University of Gothenburg, and has served on scientific advisory boards of Roche Diagnostics, Eli Lilly, and Pharmasum Therapeutics. N. Fox reports fees (paid to University College London) for consultancy from Janssen, Eli Lilly, Novartis, Sanofi, Roche/Genentech, and GlaxoSmithKline and for serving on a data monitoring committee for Biogen. Go to Neurology.org for full disclosures.

Received April 14, 2017. Accepted in final form August 21, 2017.

\section{REFERENCES}

1. Sperling RA, Aisen PS, Beckett LA, et al. Toward defining the preclinical stages of Alzheimer's disease: recommendations from the National Institute on Aging-Alzheimer's Association workgroups on diagnostic guidelines for Alzheimer's disease. Alzheimers Dement 2011;7:280-292.

2. The Ronald and Nancy Reagan Research Institute of the Alzheimer's Association and the National Institute on Aging Working Group. Consensus Report of the Working Group on Molecular and Biochemical Markers of Alzheimer's Disease. Neurobiol Aging 1998;19:109-116.

3. Olsson B, Lautner R, Andreasson U, et al. CSF and blood biomarkers for the diagnosis of Alzheimer's disease: a systematic review and meta-analysis. Lancet Neurol 2016;15: 673-684.

4. Zetterberg H, Skillback T, Mattsson N, et al. Association of cerebrospinal fluid neurofilament light concentration with Alzheimer disease progression. JAMA Neurol 2016; 73:60-67.

5. Teunissen CE, Dijkstra C, Polman C. Biological markers in CSF and blood for axonal degeneration in multiple sclerosis. Lancet Neurol 2005;4:32-41.

6. Scherling CS, Hall T, Berisha F, et al. Cerebrospinal fluid neurofilament concentration reflects disease severity in frontotemporal degeneration. Ann Neurol 2014;75: 116-126.

7. Tortelli R, Ruggieri M, Cortese R, et al. Elevated cerebrospinal fluid neurofilament light levels in patients with amyotrophic lateral sclerosis: a possible marker of disease severity and progression. Eur J Neurol 2012;19:15611567.

8. Alves S, Churlaud G, Audrain M, et al. Interleukin-2 improves amyloid pathology, synaptic failure and memory in Alzheimer's disease mice. Brain 2016;140:826-842.

9. Gaiottino J, Norgren N, Dobson R, et al. Increased neurofilament light chain blood levels in neurodegenerative neurological diseases. PLoS One 2013;8:e75091.

10. Bacioglu M, Maia LF, Preische O, et al. Neurofilament light chain in blood and CSF as marker of disease progression in mouse models and in neurodegenerative diseases. Neuron 2016;91:56-66.

11. Kuhle J, Barro C, Andreasson U, et al. Comparison of three analytical platforms for quantification of the neurofilament light chain in blood samples: ELISA, electrochemiluminescence immunoassay and Simoa. Clin Chem Lab Med 2016;54:1655-1661.

12. Rissin DM, Kan CW, Campbell TG, et al. Single-molecule enzyme-linked immunosorbent assay detects serum proteins at subfemtomolar concentrations. Nat Biotechnol 2010;28:595-599.

13. Bateman RJ, Aisen PS, De Strooper B, et al. Autosomaldominant Alzheimer's disease: a review and proposal for the prevention of Alzheimer's disease. Alzheimers Res Ther 2011;3:1.

14. Ryman DC, Acosta-Baena N, Aisen PS, et al. Symptom onset in autosomal dominant Alzheimer disease: a systematic review and meta-analysis. Neurology 2014;83: 253-260.

15. Wechsler D. Wechsler Abbreviated Scale of Intelligence (WASI). San Antonio: Psychological Corporation; 1999.

16. Nelson HE, Wilson JR. National Adult Reading Test (NART), 2nd ed. Windsor: NFER-Nelson; 1991.

17. Warrington EK. Recognition Memory Test. Windsor: NFER-Nelson; 1984.

18. Morris JC. The Clinical Dementia Rating (CDR): current version and scoring rules. Neurology 1993;43:2412-2414.

19. Rohrer JD, Woollacott IO, Dick KM, et al. Serum neurofilament light chain protein is a measure of disease intensity in frontotemporal dementia. Neurology 2016;87: 1329-1336.

20. Freeborough PA, Fox NC, Kitney RI. Interactive algorithms for the segmentation and quantitation of 3-D MRI brain scans. Comput Methods Programs Biomed 1997;53:15-25.

21. Freeborough PA, Fox NC. The boundary shift integral: an accurate and robust measure of cerebral volume changes from registered repeat MRI. IEEE Trans Med Imaging 1997;16:623-629.

22. Mattsson N, Andreasson U, Zetterberg H, Blennow K, Alzheimer's Disease Neuroimaging Initiative. Association of plasma neurofilament light with neurodegeneration in patients with Alzheimer disease. JAMA Neurol 2017;74: 557-566.

23. Jack CR Jr, Knopman DS, Jagust WJ, et al. Hypothetical model of dynamic biomarkers of the Alzheimer's pathological cascade. Lancet Neurol 2010;9:119-128.

24. Sjogren M, Blomberg M, Jonsson M, et al. Neurofilament protein in cerebrospinal fluid: a marker of white matter changes. J Neurosci Res 2001;66:510-516.

25. Weydt P, Oeckl P, Huss A, et al. Neurofilament levels as biomarkers in asymptomatic and symptomatic familial amyotrophic lateral sclerosis. Ann Neurol 2016;79: 152-158.

26. Ridha BH, Barnes J, Bartlett JW, et al. Tracking atrophy progression in familial Alzheimer's disease: a serial MRI study. Lancet Neurol 2006;5:828-834.

27. Weston PS, Nicholas JM, Lehmann M, et al. Presymptomatic cortical thinning in familial Alzheimer disease: a longitudinal MRI study. Neurology 2016;87:20502057.

28. Villemagne VL, Burnham S, Bourgeat $P$, et al. Amyloid $\beta$ deposition, neurodegeneration, and cognitive decline in sporadic Alzheimer's disease: a prospective cohort study. Lancet Neurol 2013;12:357-367.

29. Fox NC, Warrington EK, Seiffer AL, Agnew SK, Rossor MN. Presymptomatic cognitive deficits in individuals at risk of familial Alzheimer's disease. A longitudinal prospective study. Brain 1998;121:1631-1639.

30. Rojas JC, Karydas A, Bang J, et al. Plasma neurofilament light chain predicts progression in progressive supranuclear palsy. Ann Clin Transl Neurol 2016;3: 216-225. 
31. Fernandez-Martos CM, King AE, Atkinson RA, Woodhouse A, Vickers JC. Neurofilament light gene deletion exacerbates amyloid, dystrophic neurite, and synaptic pathology in the APP/PS1 transgenic model of Alzheimer's disease. Neurobiol Aging 2015;36: 2757-2767.

32. Lista S, O'Bryant SE, Blennow K, et al. Biomarkers in sporadic and familial Alzheimer's disease. J Alzheimers Dis 2015;47:291-317.

33. Zetterberg H, Wilson D, Andreasson U, et al. Plasma tau levels in Alzheimer's disease. Alzheimers Res Ther 2013; 5:9.

34. Ray S, Britschgi M, Herbert C, et al. Classification and prediction of clinical Alzheimer's diagnosis based on plasma signaling proteins. Nat Med 2007;13:1359-1362.

35. Han X, Rozen S, Boyle SH, et al. Metabolomics in early Alzheimer's disease: identification of altered plasma sphingolipidome using shotgun lipidomics. PLoS One 2011;6: e21643.
36. Booij BB, Lindahl T, Wetterberg P, et al. A gene expression pattern in blood for the early detection of Alzheimer's disease. J Alzheimers Dis 2011;23:109-119.

37. Soares HD, Chen Y, Sabbagh M, Roher A, Schrijvers E, Breteler M. Identifying early markers of Alzheimer's disease using quantitative multiplex proteomic immunoassay panels. Ann NY Acad Sci 2009;1180:56-67.

38. Mielke MM, Haughey NJ, Bandaru VV, et al. Plasma sphingomyelins are associated with cognitive progression in Alzheimer's disease. J Alzheimers Dis 2011;27: 259-269.

39. Casanova R, Varma S, Simpson B, et al. Blood metabolite markers of preclinical Alzheimer's disease in two longitudinally followed cohorts of older individuals. Alzheimers Dement 2016;12:815-822.

40. Gisslen M, Price RW, Andreasson U, et al. Plasma concentration of the neurofilament light protein (NFL) is a biomarker of CNS injury in HIV infection: a crosssectional study. EBioMedicine 2016;3:135-140.

\section{Carry the Only Card that Helps Support the AAN-and Get a \$100 Cash Rewards Bonus!}

Apply for the BankAmericard Cash Rewards ${ }^{\mathrm{TM}}$ credit card today to start getting more cash back for the things you buy most-plus a $\$ 100$ cash rewards bonus offer! Visit AAN.com/view/CashRewards and enter priority code "VACN51."

\section{Subspecialty Alerts by E-mail!}

Customize your online journal experience by signing up for e-mail alerts related to your subspecialty or area of interest. Access this free service by visiting Neurology.org/site/subscriptions/etoc.xhtml or click on the "E-mail Alerts" link on the home page. An extensive list of subspecialties, methods, and study design choices will be available for you to choose from-allowing you priority alerts to cutting-edge research in your field!

\section{Save These Dates for AAN CME Opportunities!}

Mark these dates on your calendar for exciting continuing education conferences by the American Academy of Neurology. Learn more at AAN.com/conferences.

\section{Breakthroughs in Neurology Conference}

- January 12-15, 2018, Orlando, FL, at the Caribe Royale Orlando 


\section{Neurology}

Serum neurofilament light in familial Alzheimer disease: A marker of early neurodegeneration

Philip S.J. Weston, Teresa Poole, Natalie S. Ryan, et al.

Neurology 2017;89;2167-2175 Published Online before print October 25, 2017

DOI 10.1212/WNL.0000000000004667

This information is current as of October 25, 2017

Neurology ${ }^{\circledR}$ is the official journal of the American Academy of Neurology. Published continuously since 1951, it is now a weekly with 48 issues per year. Copyright Copyright $@ 2017$ The Author(s). Published by Wolters Kluwer Health, Inc. on behalf of the American Academy of Neurology.. All rights reserved. Print ISSN: 0028-3878. Online ISSN: 1526-632X.

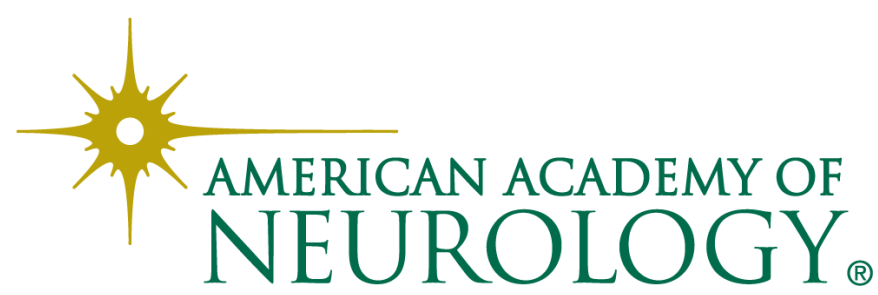




\section{Updated Information \& Services}

\section{Supplementary Material}

\section{References}

Citations

Subspecialty Collections

\section{Permissions \& Licensing}

Reprints including high resolution figures, can be found at:

http://n.neurology.org/content/89/21/2167.full

Supplementary material can be found at:

http://n.neurology.org/content/suppl/2017/10/25/WNL.0000000000004 667.DC1

http://n.neurology.org/content/suppl/2017/10/25/WNL.0000000000004 667.DC2

http://n.neurology.org/content/suppl/2018/06/28/WNL.0000000000004 667.DC3

http://n.neurology.org/content/suppl/2018/11/02/WNL.0000000000004 667.DC4

This article cites 37 articles, 4 of which you can access for free at: http://n.neurology.org/content/89/21/2167.full\#ref-list-1

This article has been cited by 4 HighWire-hosted articles: http://n.neurology.org/content/89/21/2167.full\#\#otherarticles

This article, along with others on similar topics, appears in the following collection(s):

\section{Alzheimer's disease}

http://n.neurology.org/cgi/collection/alzheimers_disease

Information about reproducing this article in parts (figures,tables) or in its entirety can be found online at:

http://www.neurology.org/about/about_the_journal\#permissions

Information about ordering reprints can be found online:

http://n.neurology.org/subscribers/advertise

Neurology ${ }^{\circledR}$ is the official journal of the American Academy of Neurology. Published continuously since 1951, it is now a weekly with 48 issues per year. Copyright Copyright ( 2017 The Author(s). Published by Wolters Kluwer Health, Inc. on behalf of the American Academy of Neurology.. All rights reserved. Print ISSN: 0028-3878. Online ISSN: 1526-632X.

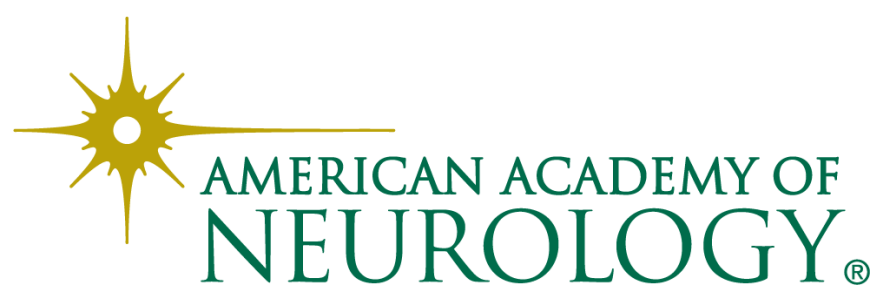

